\title{
THE OBSTACLE PROBLEM FOR THE INFINITY FRACTIONAL LAPLACIAN
}

\author{
LOURDES MORENO MÉRIDA AND RAÚL EMILIO VIDAL
}

\begin{abstract}
Given $g$ an $\alpha$-Hölder continuous function defined on the boundary of a bounded domain $\Omega$ and given $\psi$ a continuous obstacle defined in $\bar{\Omega}$, in this article, we find $u$ an $\alpha$-Hölder extension of $g$ in $\Omega$ with $u \geq \psi$. This function $u$ minimizes the $\alpha$-Hölder semi-norm of all possible extensions with these properties and it is a viscosity solution of the associated obstacle problem for the infinity fractional Laplace operator.
\end{abstract}

\section{INTRODUCTION}

Let $\Omega$ be an open, bounded domain of $\mathbb{R}^{N}$ and $\alpha \in(0,1)$. In this paper we will consider the infinite fractional Laplace operator given by

$$
L u(x)=\sup _{y \in \bar{\Omega}, y \neq x} \frac{u(y)-u(x)}{|y-x|^{\alpha}}+\inf _{y \in \bar{\Omega}, y \neq x} \frac{u(y)-u(x)}{|y-x|^{\alpha}}, \text { for } x \in \Omega .
$$

Motivated by the results of Chambolle, Lindgren and Monneau (see [6]), we will be interested in solutions of the associated Dirichlet obstacle problem. Concretely, given an $\alpha$-Hölder function $g$ defined on $\partial \Omega$ and a continuous obstacle $\psi$ defined on $\bar{\Omega}$, we aim to prove the existence and uniqueness of at least a super infinity fractional harmonic function constrained to lie above the obstacle and to take the datum on $\partial \Omega$. More precisely, we consider the following obstacle problem

$$
\begin{cases}-L u(x)=0, & \text { in } \quad\{x \in \Omega: u(x)>\psi(x)\}, \\ -L u(x) \geq 0, & \text { in } \quad\{x \in \Omega: u(x)=\psi(x)\}, \\ u(x) \geq \psi(x), & \text { if } \quad x \in \Omega, \\ u(x)=g(x), & \text { if } \quad x \in \partial \Omega,\end{cases}
$$

and we will study the existence and uniqueness of a viscosity solution that seems to be natural in this framework.

By a viscosity subsolution (resp. supersolution) of (1) we mean an upper semicontinuous (resp. lower semicontinuous) function $u$ from $\bar{\Omega}$ to $\mathbb{R}$ satisfying that $u \leq g$ (resp. $u \geq g$ ) on $\partial \Omega$ and the following property: $\forall \varphi \in C^{1}(\Omega) \cap C(\bar{\Omega})$ such that

$$
u \leq \varphi, \text { in } \bar{\Omega}(\text { resp. } u \geq \varphi),
$$

Key words and phrases. Infinity fractional Laplace operator, viscosity solutions, obstacle problem.

2010 Mathematics Subject Classification: 35D40, 35J60, 35J65. 


$$
u\left(x_{0}\right)=\varphi\left(x_{0}\right), \text { for some } x_{0} \in \Omega,
$$

then

$$
\min \left\{-L \varphi\left(x_{0}\right), \varphi\left(x_{0}\right)-\psi\left(x_{0}\right)\right\} \leq 0(\text { resp. } \geq 0) .
$$

A viscosity solution is a function which is both a subsolution and a supersolution.

We observe that, if $u$ is a continuos function defined on $\bar{\Omega}$ satisfying $u=g$ on $\partial \Omega$, then we easily deduce, from the above definition, the following characterization of viscosity sub and super solution. Concretely, $u$ is a viscosity subsolution of (1) if for any $\varphi \in C^{1}(\Omega) \cap C(\bar{\Omega})$ such that

$$
\begin{gathered}
u \leq \varphi, \text { in } \bar{\Omega}, \\
\psi\left(x_{0}\right)<u\left(x_{0}\right)=\varphi\left(x_{0}\right), \text { for some } x_{0} \in \Omega,
\end{gathered}
$$

then

$$
-L \varphi\left(x_{0}\right) \leq 0 .
$$

On the other hand, $u$ is a viscosity supersolution of (1) if for any $\varphi \in$ $C^{1}(\Omega) \cap C(\bar{\Omega})$ such that

$$
\begin{gathered}
u \geq \varphi, \text { in } \bar{\Omega}, \\
u\left(x_{0}\right)=\varphi\left(x_{0}\right), \text { for some } x_{0} \in \Omega,
\end{gathered}
$$

then

$$
-L \varphi\left(x_{0}\right) \geq 0 \quad \text { and } \quad u\left(x_{0}\right) \geq \psi\left(x_{0}\right) .
$$

It is interesting to note that the obstacle function $\psi$ is always a viscosity subsolution of (1). See [11] for more information about viscosity solutions.

We also emphasize that in order to have a solution of our obstacle problem (1), it is necessary (due to the boundary conditions) that

$$
\psi(x) \leq g(x), \forall x \in \partial \Omega,
$$

holds true.

Problem (1) involves a boundary problem in the fractional setting. This kink of problems have been studied extensively, see for instance [7] and [8]. Specifically, Problem (1) involves the infinity fractional Laplace operator The infinity Laplace operator was considered widely in the literature in the local case, see [1], [2], [5], [12] [14], and [15]; as well as in the nonlocal case (especially fractional), see for instance [4], [6] and [10]. Moreover, in [4], [12] and [15] it is studied the existence of a solution for some obstacle problems. On one hand, in [12], the authors consider the (local) infinity Laplace operator. In particular, they propose a game which involves an obstacle function and they prove that certain limit of some specific values functions is a viscosity solution of the obstacle problem for the infinity Laplacian. On the other side, in [4], the authors consider a nonlocal tug of war game. Motivated by [14] the authors consider here a nonlocal version of the game.

Recently, in [6], given an $\alpha$-Hölder continuous function $g$ defined on $\partial \Omega$, it is obtained a viscosity solution for the Dirichlet problem

$$
\begin{cases}-L u(x)=0, & \text { if } \quad x \in \Omega, \\ u(x)=g(x), & \text { if } \quad x \in \partial \Omega,\end{cases}
$$

where $L$ is the infinity fractional Laplace operator defined before. Our aim is to extend these results considering the study of the obstacle problem (1). 
Specifically, if we will denote the $\alpha$-Hölder semi-norm of a function $u$ defined on $\Omega$ by ${ }^{1}$

$$
[u]_{\alpha}=\sup _{x, y \in \Omega, x \neq y} \frac{|u(y)-u(x)|}{|y-x|^{\alpha}},
$$

our main results are the following theorems.

Theorem 1. Let $\Omega$ be an open, bounded domain of $\mathbb{R}^{N}, g \in C(\partial \Omega)$ and $\psi \in C(\bar{\Omega})$ such that $(2)$ holds true. Then there exists at most a viscosity solution $u$ of the obstacle problem (1).

Theorem 2. Let $\Omega$ be an open, bounded and Lipschitz domain of $\mathbb{R}^{N}$, $\alpha \in(0,1)$. If $g \in C^{0, \alpha}(\partial \Omega)$ and $\psi \in C(\bar{\Omega})$ satisfy (2), then there exists a unique viscosity solution $u$ of the obstacle problem (1) which belongs to $C^{0, \alpha}(\bar{\Omega})$. Moreover, the solution $u$ is the best $\alpha$-Hölder continuous extension of the datum $g$ which lies above the obstacle $\psi$, in the sense that

$$
[u]_{\alpha} \leq[z]_{\alpha},
$$

for any arbitrary $\alpha$-Hölder extension $z$ of the datum $g$ which satisfies $z \geq \psi$.

Following the arguments of [6], a possible approach to study our problem (1) is to approximate our infinity Laplace operator $L$ with a sequence of approximate operators (see Section 2 below). In this sense, in what follows, given $p>N, \frac{N}{p}<\alpha<1$ and $s:=\alpha-\frac{N}{p}$, we consider the fractional Sobolev space $W^{s, p}(\Omega)$ defined by

$$
W^{s, p}(\Omega)=\left\{u \in L^{p}(\Omega): \int_{\Omega} \int_{\Omega}\left(\frac{|u(y)-u(x)|}{|y-x|^{\alpha}}\right)^{p} d y d x<+\infty\right\},
$$

and we recall that $\left(W^{s, p}(\Omega),\|\cdot\|_{s, p}\right)$ is a Banach space, where

$$
\|u\|_{s, p}=\left(\int_{\Omega}|u|^{p}+\int_{\Omega} \int_{\Omega}\left(\frac{|u(y)-u(x)|}{|y-x|^{\alpha}}\right)^{p} d y d x\right)^{1 / p}, u \in W^{s, p}(\Omega) .
$$

We define the functional $E_{p}: W^{s, p}(\Omega) \longrightarrow \mathbb{R}$ by

$$
E_{p}(u)=\int_{\Omega} \int_{\Omega}\left(\frac{|u(y)-u(x)|}{|y-x|^{\alpha}}\right)^{p} d y d x
$$

and we study the minimization problem in a specific set. Observe that the operator of the Euler Lagrange equation associated to this functional is

$$
L_{p} u(x)=\int_{\Omega}\left(\frac{|u(y)-u(x)|}{|y-x|^{\alpha}}\right)^{p-1} \frac{\operatorname{sig}(u(y)-u(x))}{|y-x|^{\alpha}} d y,
$$

where $\operatorname{sig}(x)=\frac{x}{|x|}$ for $x \neq 0$. At least formally, we emphasize that the operator $\left(L_{p}(u)\right)^{1 /(p-1)}$ should tend to our infinity fractional Laplace operator $L$ when $p$ goes to $\infty$. We remark that this formal limit procedure only works when the right hand side is zero (when it is not zero one may expect a different limit equation). This will be the key point in our approach. We want to prove that the unique minimum (belonging to a suitable set) $u_{p}$ of $E_{p}$ is a viscosity solution of the obstacle problem associated to the operator $L_{p}$. Afterwards, we want to pass to the limit when $p$ tends to infinity. We

\footnotetext{
${ }^{1}$ Recall that $\left(C^{0, \alpha}(\Omega),\|\cdot\|_{\infty}+[.]_{\alpha}\right)$ is a Banach Space
} 
will prove that the limit of the sequence $u_{p}$ of approximate solutions is a viscosity solution of (1).

The article is organized as follows: in Section 2, we study the properties of the approximate obstacle problems (associated to the approximate operators $\left.L_{p}\right)$ and in Section 3, we prove our main results.

\section{Approximate problems}

We consider the approximate operators $L_{p}$ given by (4) and we study in this section the approximate obstacle problems

$$
\begin{cases}-L_{p} u(x)=0, & \text { in } \quad\{x \in \Omega: u(x)>\psi(x)\}, \\ -L_{p} u(x) \geq 0, & \text { in } \quad\{x \in \Omega: u(x)=\psi(x)\}, \\ u(x) \geq \psi(x), & \text { if } \quad x \in \Omega, \\ u(x)=g(x), & \text { if } \quad x \in \partial \Omega .\end{cases}
$$

In the next lemmas, we prove that the functional $E_{p}$ given by (3) has a unique minimum (in a specific set) which is a viscosity solution of the approximate obstacle problem (5).

Lemma 1. Let $\Omega$ be an open, bounded and Lipschitz domain of $\mathbb{R}^{N}, \alpha \in$ $(0,1), p>\frac{2 N}{\alpha}$. If $g \in C^{0, \alpha}(\partial \Omega), \psi \in C(\bar{\Omega})$ and (2) holds, then the functional $E_{p}$ given by (3) takes a unique minimum $u_{p}$ in the set

$$
X_{g, \psi}=\left\{v \in W^{s, p}(\Omega): v \geq \psi \text { en } \bar{\Omega}, v=g \text { en } \partial \Omega\right\} .
$$

Moreover, $u_{p}$ belongs to $C(\bar{\Omega})$.

Proof. Firstly we observe that, any $\alpha$-Hölder extension of $g$ which lies above the obstacle $\psi$ belongs to the set $X_{g, \psi}$. Thus, this set is not empty (see [15, Proposition 3.3] for the existence of this extension). In addition, if we take $u \in X_{g, \psi}$ and we fix $k \geq\|\psi\|_{\infty}+\|g\|_{\infty}$, then $T_{k}(u) \in X_{g, \psi}$ and

$$
E_{p}(u) \geq E_{p}\left(T_{k}(u)\right)
$$

where the function $T_{k}: \mathbb{R} \longrightarrow \mathbb{R}$ is defined by

$$
T_{k}(s)=\left\{\begin{array}{l}
-k, \text { si } s<-k, \\
s, \text { si }|s| \leq k \\
k, \text { si } s>k
\end{array}\right.
$$

As a consequence, we have that

$$
\min _{X_{g, \psi}} E_{p}(u)=\min _{X_{g, \psi}^{k}} E_{p}(u),
$$

where

$$
X_{g, \psi}^{k}=\left\{u \in X_{g, \psi}:\|u\|_{\infty} \leq k\right\} .
$$

Since the set $X_{g, \psi}^{k}$ is weakly closed (with the weak topology of $W^{s, p}(\Omega)$ ), to prove the existence of a minimum in this set, we will study the coercivity and the weak lower semicontinuity of the functional $E_{p}$.

On the one hand, we take a sequence $\left\{u_{n}\right\} \subset X_{g, \psi}^{k}$ such that $\left\|u_{n}\right\|_{s, p} \rightarrow$ $+\infty$. Since $\left\|u_{n}\right\|_{\infty} \leq k$, this necessarily means that $E_{p}\left(u_{n}\right) \rightarrow+\infty$. That is, our functional is coercive. 
On the other hand, we take a sequence $\left\{u_{n}\right\} \subset X_{g, \psi}^{k}$ such that $u_{n}$ weakly converges to a function $u$ in $W^{s, p}(\Omega)$. Since $W^{s, p}(\Omega)$ is compactly embedded in $L^{p}(\Omega)$ (see $\left[9\right.$, Corollary 1.2]) and the norm $\|.\|_{s, p}$ is a w.l.s.c. function, then

as we desired.

$$
\liminf E_{p}\left(u_{n}\right) \geq E_{p}(u)
$$

Consequently, the functional $E_{p}$ has a minimum $u_{p}$ in the set $X_{g, \psi}$ and again by $\left[9\right.$, Theorem 8.2], $u_{p} \in C(\bar{\Omega})$. Moreover, since the functional is convex, this minimum is unique.

Lemma 2. Let $\Omega$ be an open, bounded and Lipschitz domain of $\mathbb{R}^{N}, g \in$ $C^{0, \alpha}(\partial \Omega)$ and $\psi \in C(\bar{\Omega})$ satisfying (2). If $p>2 N / \alpha$, then the minimum $u_{p}$ (given by Lemma 1) is a viscosity solution of (5).

Proof. Firstly, recall that the minimum $u_{p}$ (given by Lemma 1 ) belongs to $C(\bar{\Omega})$ and satisfies $u_{p}=g$ on $\partial \Omega$ and $u_{p} \geq \psi$ in $\bar{\Omega}$. We will prove that $u_{p}$ is a viscosity sub and super solution. On the one hand, we claim that $u_{p}$ is a viscosity subsolution of (5). Indeed, we take $\varphi \in C^{1}(\Omega) \cap C(\bar{\Omega})$ such that

$$
\begin{gathered}
u_{p} \leq \varphi, \text { in } \bar{\Omega}, \\
\psi\left(x_{0}\right)<u_{p}\left(x_{0}\right)=\varphi\left(x_{0}\right), \text { for some } x_{0} \in \Omega,
\end{gathered}
$$

and we prove $-L \varphi\left(x_{0}\right) \leq 0$. Without loss of generality, we suppose that $\varphi$ touches $u_{p}$ only at the point $x_{0}$; otherwise it is sufficient to replace $\varphi$ by $\varphi(x)+\delta\left|x-x_{0}\right|^{2}$ with $\delta$ small enough. We define the functions

$$
\varphi^{\epsilon}=\max \left(u_{p}, \varphi-\epsilon\right),
$$

and

$$
\varphi_{\epsilon}=\min \left(u_{p}, \varphi-\epsilon\right)
$$

Since, we suppose that $\varphi\left(x_{0}\right)=u_{p}\left(x_{0}\right)>\psi\left(x_{0}\right)$, for $\epsilon$ small enough, $\varphi_{\epsilon} \geq \psi$ in $\Omega$ and moreover, $\varphi_{\epsilon}=u_{p}$ on $\partial \Omega$. Hence, $\varphi_{\epsilon}$ belongs to $X_{g, \psi}$ and using that $u_{p}$ is a minimum of $E_{p}$ in this set, we have

$$
E_{p}\left(\varphi_{\epsilon}\right) \geq E_{p}\left(u_{p}\right) .
$$

From this inequality and using the following convexity inequality (see $[6$, Lemma 6.2])

$$
|\max (a, c)-\max (b, d)|^{p}+|\min (a, c)-\min (b, d)|^{p} \leq|a-b|^{p}+|c-d|^{p},
$$

for all $p \geq 1$, we deduce that

$$
E_{p}\left(\varphi_{\epsilon}\right)+E_{p}\left(\varphi^{\epsilon}\right) \leq E_{p}\left(u_{p}\right)+E_{p}(\varphi) \leq E_{p}\left(\varphi_{\epsilon}\right)+E_{p}(\varphi),
$$

that is

$$
E_{p}\left(\varphi^{\epsilon}\right) \leq E_{p}(\varphi) .
$$

The convexity of $E_{p}$ implies

$$
E_{p}\left((1-t) \varphi+t \varphi^{\epsilon}\right) \leq(1-t) E_{p}(\varphi)+t E_{p}\left(\varphi^{\epsilon}\right) \leq E_{p}(\varphi),
$$

and then we have

$$
\frac{E_{p}\left((1-t) \varphi+t \varphi^{\epsilon}\right)-E_{p}(\varphi)}{t} \leq 0 .
$$

Let call

$$
f(t)=E_{p}\left((1-t) \varphi+t \varphi^{\epsilon}\right) .
$$


From the above inequality and using the convexity of the function $f$, we have

and then

$$
f^{\prime}(0) \leq \frac{f(t)-f(0)}{t} \leq 0,
$$

where

$$
p \int_{\Omega} \int_{\Omega} H(x, y) d y d x \leq 0
$$

$H(x, y)=\left|\frac{\varphi(y)-\varphi(x)}{|y-x|^{\alpha}}\right|^{p-1} \frac{\operatorname{sgn}(\varphi(y)-\varphi(x))}{|y-x|^{\alpha}}\left(\varphi^{\epsilon}(y)-\varphi(y)+\epsilon-\varphi^{\epsilon}(x)+\varphi(x)-\epsilon\right)$.

Therefore, a change of variable implies

$$
\int_{\Omega}\left(\varphi^{\epsilon}-\varphi+\epsilon\right)(x)\left(-L_{p} \varphi(x)\right) d x \leq 0 .
$$

Now we argue by contradiction. Suppose that $-L_{p} \varphi\left(x_{0}\right)>0$. By continuity, which holds under our assumptions, there is a small ball $B_{r}\left(x_{0}\right)$ such that $-L_{p} \varphi>0$ in $B_{r}\left(x_{0}\right)$. Since $\varphi^{\epsilon}=\max \left(u_{p}, \varphi-\epsilon\right)$, for $\epsilon$ small enough, we have $\operatorname{supp}\left(\varphi^{\epsilon}-\varphi+\epsilon\right) \subset B_{r}\left(x_{0}\right)$. We also observe that $\varphi^{\epsilon}-\varphi+\epsilon \geq 0$. Consequently, we deduce

$$
0<\int_{B_{r}\left(x_{0}\right)}\left(\varphi^{\epsilon}-\varphi+\epsilon\right)(x)\left(-L_{p} \varphi(x)\right) d x \leq 0
$$

which is a contradiction.

In the same way, one can prove that $u$ is a viscosity supersolution.

\section{Proofs of the main Results}

Proof of Theorem 1. Suppose that $u$ and $v$ are two viscosity solutions of the obstacle problem (1) and define the set

$$
W=\{x \in \Omega: u(x)>v(x)\} .
$$

We claim that $W$ is an empty set. Indeed, arguing by contradiction, we suppose that $W$ is not empty. Since $v \geq \psi$ in $\Omega$, we have $v \geq \psi$ and $u>\psi$ in $W$. Consequently, the functions $u$ and $v$ satisfy

$$
\left\{\begin{array} { l } 
{ - L u = 0 \quad \text { in } W , } \\
{ u = u \quad \text { on } \partial W , }
\end{array} \quad \left\{\begin{array}{l}
-L v \geq 0 \quad \text { in } W, \\
v=u \quad \text { on } \partial W,
\end{array}\right.\right.
$$

which implies, using the comparison principle [6, Proposition 11.2], that $v \geq u$ in $W$. This is a contradiction and the claim is proved. Reversing the role of $u$ and $v$ gives that $u=v$ which conclude the proof.

To prove Theorem 2 we need the following technical result.

Lemma 3. [6, Lemma 6.5] For $\varphi \in C^{1}(\Omega), p \geq 1$ and $\alpha \in(0,1)$, we define

$$
f_{p}(y)=\frac{\varphi(y)-\varphi\left(x_{p}\right)}{\left|y-x_{p}\right|^{\alpha}} \quad \text { and } \quad f(y)=\frac{\varphi(y)-\varphi\left(x_{0}\right)}{\left|y-x_{0}\right|^{\alpha}},
$$

where $x_{p} \longrightarrow x_{0} \in \Omega$ as $p \longrightarrow \infty$. Then,

$$
\lim _{p \longrightarrow \infty}\left\|\frac{f_{p}^{+}(y)}{\left|y-x_{p}\right|^{\alpha / p}}\right\|_{L^{p}(\Omega)}=\left\|f^{+}\right\|_{L^{\infty}(\Omega)},
$$


with $f^{ \pm}(x)=\max ( \pm f(x), 0)$. The same also holds for $f_{p}^{-}$.

Proof of Theorem 2. Let $\left\{u_{p}\right\}$ be a sequence of viscosity solutions $u_{p}$ of (5) given by Lemma 1. Our aim is to pass to the limit when $p$ goes to infinite. Firstly, given $\alpha \in(0,1)$, we prove that the sequence $\left\{u_{p}\right\}$ is bounded in $W^{s, q}(\Omega)$ for any $q>2 N / \alpha$. Indeed, by construction, there is a positive constant $k$ such that

$$
\left\|u_{p}\right\|_{\infty} \leq k, \forall p
$$

Now, we take any $p>2 N / \alpha$ and we fix a number $q$ such that $2 N / \alpha<q<p$. Let $z$ be a Hölder extension of $g$ such that $z \geq \psi$ (see [15, Proposition 3.3]). Since the functional $E_{p}$ takes a unique minimum $u_{p}$ in the set $X_{g, \psi}$, then

$$
E_{p}\left(u_{p}\right) \leq E_{p}(z) \leq|\Omega|^{2}[z]_{\alpha}^{p},
$$

and by Hölder inequality

$$
E_{q}\left(u_{p}\right) \leq E_{p}\left(u_{p}\right)^{\frac{q}{p}}|\Omega|^{\frac{2(p-q)}{p}} \leq|\Omega|^{2}[z]_{\alpha}^{q},
$$

which implies that the sequence $\left\{u_{p}\right\}$ is bounded in $W^{s, q}(\Omega)$. By the Sobolev embedding (see [9, Theorem 8.2]) we deduce that, up to a subsequence, $u_{p}$ strongly converges to a function $u$ in $C(\bar{\Omega})$. Moreover, since $u_{p}=g$ on $\partial \Omega$, and $u_{p} \geq \psi$ in $\Omega$, then we also have that the function $u$ satisfies

$$
u=g \text {, on } \partial \Omega, u \geq \psi, \text { in } \Omega .
$$

Now, we will prove that $u$ is a viscosity sub and super solution of problem (1). On the one hand, we claim that $u$ is a viscosity subsolution. Indeed, we take $\varphi \in C^{1}(\Omega) \cap C(\bar{\Omega})$ such that

$$
\begin{gathered}
u \leq \varphi, \text { in } \bar{\Omega}, \\
\psi\left(x_{0}\right)<u\left(x_{0}\right)=\varphi\left(x_{0}\right), \text { for some } x_{0} \in \Omega,
\end{gathered}
$$

and, without loss of generality, we suppose that $\varphi$ touches $u$ only at the point $x_{0}\left(x_{0}\right.$ is a strict maximum of $\left.u-\varphi\right)$. Hence,

$$
M_{p}:=\sup _{\bar{\Omega}}\left(u_{p}-\varphi\right)=\left(u_{p}-\varphi\right)\left(x_{p}\right),
$$

where

$$
x_{p} \mapsto x_{0}, \quad M_{p} \mapsto 0 .
$$

Moreover, since $\varphi\left(x_{0}\right)>\psi\left(x_{0}\right)$, we can suppose that $\varphi\left(x_{p}\right)>\psi\left(x_{p}\right)$ for $p$ large enough. This shows that

$$
\left\{\begin{array}{l}
u_{p} \leq \varphi_{p}:=\varphi+M_{p} \\
\psi\left(x_{p}\right)<u_{p}\left(x_{p}\right)=\varphi\left(x_{p}\right) .
\end{array}\right.
$$

The fact that $u_{p}$ is a viscosity solution implies

$$
0 \geq-L_{p} \varphi_{p}\left(x_{p}\right)=-L_{p} \varphi\left(x_{p}\right),
$$

that is,

$$
0 \geq-\int_{\Omega}\left|\frac{\varphi(y)-\varphi\left(x_{p}\right)}{\left|y-x_{p}\right|^{\alpha}}\right|^{p-1} \frac{\operatorname{sgn}\left(\varphi(y)-\varphi\left(x_{p}\right)\right)}{\left|y-x_{p}\right|^{\alpha}} d y,
$$

or equivalently,

$$
\left\|\left(\frac{\varphi(y)-\varphi\left(x_{p}\right)}{\left|y-x_{p}\right|^{\alpha+\frac{\alpha}{p-1}}}\right)^{+}\right\|_{L^{p-1}(\Omega)} \geq\left\|\left(\frac{\varphi(y)-\varphi\left(x_{p}\right)}{\left|y-x_{p}\right|^{\alpha+\frac{\alpha}{p-1}}}\right)^{-}\right\|_{L^{p-1}(\Omega)} .
$$


Thanks to Lemma 3, we can pass to the limit in this inequality to obtain

$$
\sup _{y \in \Omega}\left(\max \left(\frac{\varphi(y)-\varphi\left(x_{0}\right)}{\left|y-x_{0}\right|^{\alpha}}, 0\right)\right)+\inf _{y \in \Omega}\left(\min \left(\frac{\varphi(y)-\varphi\left(x_{0}\right)}{\left|y-x_{0}\right| \alpha}, 0\right)\right) \geq 0 .
$$

Since $\varphi$ is $C^{1}$ at $x_{0}$, it is clear that $L^{+} \varphi\left(x_{0}\right) \geq 0$ and $L^{-} \varphi\left(x_{0}\right) \leq 0$, where

$$
L^{+} \varphi\left(x_{0}\right)=\sup _{y \in \Omega, y \neq x_{0}} \frac{\varphi(y)-\varphi\left(x_{0}\right)}{\left|y-x_{0}\right|^{\alpha}}, \quad L^{-} \varphi\left(x_{0}\right)=\inf _{y \in \Omega, y \neq x_{0}} \frac{\varphi(y)-\varphi\left(x_{0}\right)}{\left|y-x_{0}\right|^{\alpha}} .
$$

Summing up, we deduce

$$
-L \varphi\left(x_{0}\right) \leq 0,
$$

as we desired.

Finally, in the same way, one can proved that $u$ is a viscosity super solution. Therefore, $u$ is a viscosity solution of the obstacle problem (1).

To conclude, we characterize the function limit $u$. In order to do it, let $z$ be any Hölder extension of $g$ such that $z \geq \psi$. By (6), we have

$$
E_{q}\left(u_{p}\right) \leq|\Omega|^{2}[z]_{\alpha}^{q},
$$

which implies, passing to the limit as $p$ goes to $\infty$,

$$
\left(E_{q}(u)\right)^{1 / q} \leq|\Omega|^{2 / q}[z]_{\alpha} .
$$

As a consequence, when $q$ tends to $\infty$, we obtain

$$
\left\|\frac{u(y)-u(x)}{|y-x|^{\alpha}}\right\|_{L^{\infty}(\Omega \times \Omega)} \leq[z]_{\alpha},
$$

i.e., we have proved that $u \in C^{0, \alpha}(\bar{\Omega})$ and moreover

$$
[u]_{\alpha} \leq[z]_{\alpha},
$$

for any arbitrary Hölder extension $z$ of the datum $g$ which satisfies $z \geq \psi$, as we desired.

Acknowledgements. The first author was supported by MINECO-FEDER grant MTM2015-68210-P, Junta de Andalucía FQM-116 and Ministerio de Educación, Cultura y Deporte (Spain) FPU grant FPU12/02395. The second author was partially supported by IEMath-Granada, CONICET and Secyt-UNC.

\section{REFERENCES}

[1] Aronsson, G. On certain singular solutions of the partial differential equation $u_{x}^{2} u_{x x}+$ $2 u_{x} u_{y} u_{x y}+u_{y}^{2} u_{y y}=0$. Manuscripta mathematica, vol. 47, no 1-3, (1984), p. 133-151.

[2] Aronsson, G., Crandall, M., and Juutinen, P. A tour of the theory of absolutely minimizing functions. Bulletin of the American mathematical society, vol. 41, no 4, (2004), p. 439-505.

[3] Barles, G.; Chasseigne, E. and Imbert, C. On the Dirichlet problem for second-order elliptic integro-differential equations. Indiana University Mathematics Journal, vol. 57, no $1,(2008)$, p. 213-146.

[4] Bjorland C., Caffarelli L. and Figalli A. Nonlocal TugofWar and the Infinity Fractional Laplacian. Communications on Pure and Applied Mathematics, vol. 65, no 3, (2012), p. 337-380.

[5] Bhattacharya, T.; Dibenedetto, E. and Manfredi, J. Limits as $p \rightarrow \infty$ of $\Delta_{p} u_{p}=f$ and related extremal problems. Rend. Sem. Mat. Univ. Pol. Torino, Fascicolo Speciale Nonlinear PDEs, (1989), p. 15-68. 
[6] Chambolle, A., Lindgren, E. and Monneau, R. A Hölder infinity Laplacian. Optimisation and Calculus of Variations, vol. 18, no 03, (2012), p. 799-835.

[7] Caffarelli, L., Salsa, S., and Silvestre, L. Regularity estimates for the solution and the free boundary of the obstacle problem for the fractional Laplacian. Inventiones mathematicae, vol 171, no 2, (2008), p. 425-461.

[8] Dipierro, S., Savin, O., and Valdinoci, E. A nonlocal free boundary problem. SIAM Journal on Mathematical Analysis, vol. 47, no 6, (2015), p. 4559-4605.

[9] Di Nezza, E.; Palatucci, G. and Valdinoci, E. Hitchhikers guide to the fractional Sobolev spaces. Bulletin des Sciences Mathmatiques. vol. 136, no 5, (2012), p. 521-573.

[10] Ferreira, R. and Pérez-Llanos, M. Limit problems for a fractional p-Laplacian as $p \rightarrow \infty$. Nonlinear Differ. Equ. Appl. no 2, Art. 14, 28pp., (2016). doi:10.1007/s00030016-0368-z

[11] Lions Crandall, M.; Ishit, H. and Lions, P. Users guide to viscosity solutions of second order partial differential equations. Bulletin of the American Mathematical Society, vol. 27, no 1, (1992), p. 1-67.

[12] Manfredi, J.; Rossi, J. and Somersille, S. An obstacle problem for Tug-of-War games. Communications on Pure and Applied Analysis. Vol. 14(1), (2015), p. 217-228.

[13] Mcshne, E. Extension of range of functions. Bulletin of the American Mathematical Society, vol. 40, no 12, (1934), p. 837-842.

[14] Peres, Y., Schramm, O., Sheffield, S. and Wilson, D. Tug-of-war and the infinity Laplacian. Journal of the American Mathematical Society, vol. 22, no 1, (2009), p. $167-210$.

[15] Rossi, J.; Teixeira, E. and Urbano J. Optimal regularity at the free boundary for the infinity obstacle problem. Interfaces and Free Boundaries. Vol. 17(3), (2015), p. 381-398.

(L. Moreno-Mérida)

Universidad de Granada, Granada, España.

EMAIL: lumore@ugr.es

(R. E. Vidal)

FaMAF, Universidad Nacional de Cordoba, (5000), Cordoba, Argentina.

EMAIL: vidal@mate.uncor.edu 